\title{
Multilocal Fermionization
}

\author{
KARL-HENNING REHREN ${ }^{1,2}$ and GENNARO TEDESCO ${ }^{1}$ \\ ${ }^{1}$ Institut für Theoretische Physik, Universität Göttingen, Friedrich-Hund-Platz 1, \\ 37077 Göttingen, Germany. e-mail: rehren@theorie.physik.uni-goettingen.de \\ ${ }^{2}$ Courant Research Centre "Higher Order Structures in Mathematics", \\ Universität Göttingen, Bunsenstr. 3-5, 37073 Göttingen, Germany
}

Received: 8 May 2012 / Revised: 26 July 2012 / Accepted: 13 August 2012

Published online: 1 September 2012 - (C) The Author(s) 2012. This article is published with open access at Springerlink.com

\begin{abstract}
We present a simple isomorphism between the algebra of one real chiral Fermi field and the algebra of $n$ real chiral Fermi fields. The isomorphism preserves the vacuum state. This is possible by a "change of localization", and gives rise to new multilocal symmetries generated by the corresponding multilocal current and stress-energy tensor. The result gives a common underlying explanation of several remarkable recent results on the representation of the free Bose field in terms of free Fermi fields (Anguelova, arXiv:1112.3913, 2011; Anguelova, arXiv:1206.4026, 2012), and on the modular theory of the free Fermi algebra in disjoint intervals (Casini and Huerta, Class Quant Grav 26:185005, 2009; Longo et al., Rev Math Phys 22:331-354, 2010)
\end{abstract}

Mathematics Subject Classification. 81T40.

Keywords. CAR algebra, conformal field theory, modular theory.

\section{Introduction}

It is well known that there is an algebraic isomorphism (the "split isomorphism") $A(x) B(y) \mapsto A(x) \otimes^{t} B(y), x \in O_{1}, y \in O_{2}$ between the fields of a given QFT localized in two spacetime regions $O_{i}$, and two independent copies of the same fields localized in the same regions, as long as the regions are sufficiently well space-like separated from each other. ${ }^{1}$

Naively, this is true because $A$ (anti)commute with $B$ by graded locality, while $A \otimes^{t} \mathbf{1}$ (anti)commute with $1 \otimes^{t} B$ by construction, so that all algebraic relations are preserved. However, this isomorphism does not preserve the vacuum state, because it eliminates all correlations between fields in $O_{1}$ and fields in $O_{2}$ : $\omega\left(A(x) \otimes^{t} B(y)\right)=\omega(A(x)) \cdot \omega(B(y)) \neq \omega(A(x) B(y))$, nor is it an isomorphism

\footnotetext{
${ }^{1}$ The graded tensor product $A \otimes \otimes^{t} B$ is the true tensor product $A \otimes B$ if $A$ is a Bose field, and a twisted tensor product $A \otimes(-1)^{F} B$ if $A$ is a Fermi field, such that $1 \otimes^{t} B$ and $A \otimes^{t} \mathbf{1}$ anticommute when $A$ and $B$ are both Fermi fields.
} 
of topological algebras. Moreover, it cannot be defined globally, because the restriction to space-like separated regions is essential in the argument.

We present here a new isomorphism between the algebra of one real chiral Fermi field, and the algebra of a complex $=$ two real chiral Fermi fields (or actually of any number $n$ of real Fermi fields), which preserves the vacuum state and is globally defined. The price to pay is a change of the notion of localization. This means in particular, that the isomorphism does not intertwine the respective conformal transformations. We shall discuss the relation between the stress-energy tensors of the real Fermi field and of the complex Fermi field, and features of the "embedded" diffeomorphisms generated by either SET acting on the other field. We shall also present the bilocal gauge transformations of the real Fermi field generated by the current of the complex Fermi field. This isomorphism provides a very simple understanding of two recent remarkable results: The first is the fact [2] that besides the standard fermionization formula $j(x)=: \phi^{*}(x) \phi(x)$ : of the free chiral Bose current in terms of a complex Fermi field, one can obtain the same current as a bilocal Wick product of a real Fermi field at two different points: $j(x) \sim$ $: \psi\left(x_{1}\right) \psi\left(x_{2}\right)$ : , where $x_{2}=-1 / x_{1}$ are the two solutions of $q\left(x_{i}\right) \equiv 2 x_{i} /\left(1-x_{i}^{2}\right)=x$. (The differential algebra underlying this relation was described much earlier in [8] in the context of hierarchies of integrable systems.) The isomorphism generalizes to an isomorphism of one real Fermi field with any number $n$ of real Fermi fields (at the price of an $n$-fold localization). Since non-abelian current algebras can be constructed as certain subalgebras of $n$-free-Fermi quantum field theories, it would be interesting to study the resulting multilocal embedding into the theory of a single free Fermi field. The second remarkable recent result is the discovery [7] (see also [15]) that the modular automorphism group of the local von Neumann algebra of a free Fermi theory in a union of disjoint intervals in the vacuum state acts "almost" geometric. The geometric part of the "modular dynamics" is given by the pullback of the dilation group under a rational uniformizing function that maps each of the intervals onto $\mathbb{R}_{+}$and each component of the complement onto $\mathbb{R}_{-}$; in addition, there occurs a "mixing" among the fields at related points in each of the intervals. Under the present isomorphism, the $n$ Fermi fields at a point $X$ are mapped to position-dependent linear combinations of a single Fermi field at the $n$ pre-images $x_{k}$ of $X$. Because the isomorphism preserves the vacuum state, it intertwines the respective modular automorphisms. Therefore, the modular mixing of one Fermi field in $n$ intervals is naturally explained from the well-known modular automorphism group of $n$ independent Fermi fields in a single interval, which is just the subgroup of the Möbius group preserving the interval [5,11].

Remark. With the term "fermionization", we refer to the representation of Bose fields in terms of Fermi fields, as the opposite of "bosonization", the representation of Fermi fields in terms of Bose fields $[6,16,17]$. 


\section{The Starting Point}

Let $\psi$ be the real chiral Fermi field: $\psi(x)^{*}=\psi(x)$, with canonical anticommutation relations (CAR)

$$
\{\psi(x), \psi(y)\}=2 \pi \delta(x-y), \quad(x, y \in \mathbb{R})
$$

and vacuum two-point function

$$
\omega(\psi(x) \psi(y))=\frac{-i}{x-y} \equiv \lim _{\varepsilon \searrow 0} \frac{-i}{x-y-i \varepsilon} .
$$

Let $\phi$ be a complex chiral Fermi field: $\phi(x)^{*}=\phi^{*}(x)$, with CAR

$$
\left\{\phi(x), \phi^{*}(y)\right\}=\left\{\phi^{*}(x), \phi(y)\right\}=2 \pi \delta(x-y), \quad\{\phi, \phi\}=\left\{\phi^{*}, \phi^{*}\right\}=0
$$

and vacuum two-point function

$$
\omega\left(\phi(x) \phi^{*}(y)\right)=\omega\left(\phi^{*}(x) \phi(y)\right)=\lim _{\varepsilon \searrow 0} \frac{-i}{x-y-i \varepsilon}, \quad \omega(\phi \phi)=\omega\left(\phi^{*} \phi^{*}\right)=0 .
$$

The complex Fermi field can be decomposed into two anti-commuting real Fermi fields:

$$
\phi(x)=\left(\psi^{(1)}(x)+i \psi^{(2)}(x)\right) / \sqrt{2} .
$$

We write CAR and $\mathrm{CAR}^{n}$ for the algebras generated by one, resp. $n$ real Fermi fields.

Let us also introduce the "compact picture", using the Cayley transformation

$$
z=\frac{1+i x}{1-i x} \in S^{1} \backslash\{-1\}
$$

and the definition

$$
\widehat{\psi}(z):=\left(-i \frac{\mathrm{d} z}{\mathrm{~d} x}\right)^{-\frac{1}{2}} \psi(x) \equiv \frac{1-i x}{\sqrt{2}} \psi(x)
$$

and likewise for all other Fermi fields. ${ }^{2}$ Then, for the real field, one has $\widehat{\psi}(z)^{*}=$ $z \widehat{\psi}(z)$, and for the complex field $\widehat{\phi}(z)^{*}=z \widehat{\phi}^{*}(z)$. The non-vanishing two-point functions are

$$
\omega(\widehat{\psi}(z) \widehat{\psi}(w))=\omega\left(\widehat{\phi}(z) \widehat{\phi}^{*}(w)\right)=\omega\left(\widehat{\phi^{*}}(z) \widehat{\phi}(w)\right)=\frac{1}{z-w} \equiv \lim _{\lambda \nearrow 1} \frac{1}{z-\lambda w},
$$

and the CAR is given in terms of $\lim _{\lambda{ }_{1}}\left(\frac{1}{z-\lambda w}+\frac{1}{w-\lambda z}\right)=2 \pi \delta(z, w) \equiv \frac{2 \pi}{z} \delta(\varphi-\vartheta)$ for $z=e^{i \varphi}, w=e^{i \vartheta}, \varphi, \vartheta \in(-\pi, \pi)$.

The "compact picture" CAR algebras on the cut circle $S^{1} \backslash\{-1\}$ are just reparametrizations of the CAR algebras on $\mathbb{R}$. Their extension to the full circle

\footnotetext{
${ }^{2}$ The present choice of the branch of the square root in the transformation law determines the branch of similar square roots throughout.
} 
$S^{1}$ depends on the representation. The CAR algebra on $\mathbb{R}$ possesses two faithful representations: the vacuum (or Neveu-Schwarz) representation and the Ramond representation [10]. The former extends periodically to $S^{1}$, while the latter extends anti-periodically to $S^{1}$, i.e., it extends to the twofold covering of $S^{1}$.

Our starting point (Proposition 1) is an isomorphism between the complex Fermi algebra $\operatorname{CAR}^{2}\left(S^{1}\right)$ and the real Fermi algebra $\operatorname{CAR}\left(S^{1}\right)$ in the vacuum representation. We shall give two simple proofs. The simplest one is in the global setting of the Fourier modes. We also give another, local proof, because our main interest in the rest of the paper lies in the local properties of the resulting new symmetries of the real Fermi field, which can be understood by this isomorphism. These symmetries are in a controlled way non-local ("bilocal"), If they were completely non-local, there would be little interest; we believe, however, that the bilocal symmetries are of some physical relevance. This will be elaborated in Section 4, where contact with the modular theory of the real Fermi field is made.

Due to the different global behaviour, the following proposition holds only in the vacuum representation. It entails bilocal fermionization formulae for the current and the stress-energy tensor in the vacuum representation (Corollaries 1 and 3). An analogue of the proposition in the Ramond sector will be discussed in Section 5.

PROPOSITION 1. Let $\phi$ and $\psi$ stand for the complex and real Fermi fields in the vacuum representation. The linear map

$$
\begin{aligned}
\beta: \widehat{\phi}\left(z^{2}\right) & \mapsto \frac{1}{2}(\widehat{\psi}(z)+\widehat{\psi}(-z)), \\
\widehat{\phi}^{*}\left(z^{2}\right) & \mapsto \frac{1}{2 z}(\widehat{\psi}(z)-\widehat{\psi}(-z))
\end{aligned}
$$

for $z \in S^{1}$, induces an isomorphism $\beta: \mathrm{CAR}^{2}\left(S^{1}\right) \rightarrow \mathrm{CAR}\left(S^{1}\right)$ of $C A R$ algebras, which preserves the vacuum state.

Note that the map is well defined because the right-hand sides are invariant under $z \leftrightarrow-z$.

Proof. The simplest proof proceeds by looking at the Fourier modes of the real and complex free Fermi fields,

$$
\widehat{\psi}(z)=\sum_{n \in \mathbb{Z}+\frac{1}{2}} \psi_{n} z^{-n-\frac{1}{2}}, \quad \widehat{\phi}(z)=\sum_{n \in \mathbb{Z}+\frac{1}{2}} \phi_{n} z^{-n-\frac{1}{2}}, \quad \widehat{\phi}^{*}(z)=\sum_{n \in \mathbb{Z}+\frac{1}{2}}\left(\phi^{*}\right)_{n} z^{-n-\frac{1}{2}},
$$

where $\psi_{n}=\left(\psi_{-n}\right)^{*}$ and $\left(\phi^{*}\right)_{n}=\left(\phi_{-n}\right)^{*}$ satisfy the CAR $\left\{\psi_{n}, \psi_{m}\right\}=\delta_{n+m, 0},\left\{\phi_{n}, \phi_{m}^{*}\right\}=$ $\delta_{n+m, 0}$. In terms of these modes, the isomorphism is given by

$$
\beta\left(\widehat{\phi}\left(z^{2}\right)\right)=\sum_{n \in \mathbb{Z}+\frac{1}{2}} \psi_{2 n+\frac{1}{2}}\left(z^{2}\right)^{-n-\frac{1}{2}}, \quad \beta\left(\widehat{\phi}^{*}\left(z^{2}\right)\right)=\sum_{n \in \mathbb{Z}+\frac{1}{2}} \psi_{2 n-\frac{1}{2}}\left(z^{2}\right)^{-n-\frac{1}{2}} .
$$


This is just a renumbering of the generators

$$
\phi_{n} \mapsto \psi_{2 n+\frac{1}{2}}, \quad\left(\phi^{*}\right)_{n} \mapsto \psi_{2 n-\frac{1}{2}} \quad\left(n \in \mathbb{Z}+\frac{1}{2}\right),
$$

such that the conjugate complex fields $\beta(\widehat{\phi}), \beta\left(\widehat{\phi}^{*}\right)$ involve the Fourier modes $\psi_{m}$ of the real Fermi field with $m \in 2 \mathbb{Z}-\frac{1}{2}, m \in 2 \mathbb{Z}+\frac{1}{2}$, respectively.

It is then obvious that the CAR and adjoint relations are preserved. The vacuum state is preserved because the vacuum is the unique state which is annihilated by all $\psi_{n}(n>0)$, and by all $\phi_{n}, \phi_{n}^{*}(n>0)$, respectively. This already proves the statement.

We wish, however, to emphasize the local aspects of the isomorphism. Therefore, we also indicate the proof in the setting of local quantum field theory.

One may directly compute the anti-commutators

$$
\left\{\beta\left(\widehat{\phi}^{*}\left(z^{2}\right)\right), \beta\left(\widehat{\phi}\left(w^{2}\right)\right)\right\}=2 \pi \delta\left(z^{2}, w^{2}\right)=\frac{\pi}{z}(\delta(z, w)+\delta(z,-w)),
$$

etc. It is, however, sufficient and in fact much easier, to verify the equality of the vacuum two-point functions

$$
\omega\left(\beta\left(\widehat{\phi^{*}}\left(z^{2}\right)\right) \beta\left(\widehat{\phi}\left(w^{2}\right)\right)\right)=\frac{1}{z^{2}-w^{2}},
$$

etc, which is easily done by a direct computation. By Wick's theorem, this equality then extends to all $n$-point functions. The CAR follow from these correlation functions.

The adjoint relation

$$
\beta\left(\widehat{\phi}\left(z^{2}\right)\right)^{*}=z^{2} \beta\left(\widehat{\phi}^{*}\left(z^{2}\right)\right)
$$

is immediate. Thus $\beta$ is a ${ }^{*}$-homomorphism.

Finally, $\beta$ is an isomorphism because it has an inverse:

$$
\beta^{-1}(\widehat{\psi}(z))=\widehat{\phi}\left(z^{2}\right)+z \widehat{\phi}^{*}\left(z^{2}\right) .
$$

This proves the proposition.

Going back to the non-compact picture, the isomorphism can be written as

$$
\begin{aligned}
\phi(q(x)) & \mapsto \frac{1}{q(x)}\left[\frac{x \psi(x)}{1-i x}+\frac{i \psi\left(-\frac{1}{x}\right)}{1+i x}\right], \\
\phi^{*}(q(x)) & \mapsto \frac{1}{q(x)}\left[\frac{x \psi(x)}{1+i x}-\frac{i \psi\left(-\frac{1}{x}\right)}{1-i x}\right],
\end{aligned}
$$

where $q(x)=\frac{2 x}{1-x^{2}}$ is the map $(-1,1) \rightarrow \mathbb{R}$ corresponding, under the Cayley transformation, to the square map on $z \in S^{1}$. The inverse reads

$$
\beta^{-1}(\psi(x))=\frac{1+i x}{1-x^{2}} \phi(q(x))+\frac{1-i x}{1-x^{2}} \phi^{*}(q(x)) .
$$


Equipped with this isomorphism, we shall discuss several implications in the sequel of this paper:

1. Under this isomorphism, the standard local fermionization formula for the current in terms of the complex CAR algebra

$$
\widehat{j}(z)=: \widehat{\phi^{*}}(z) \widehat{\phi}(z):=i: \widehat{\psi^{(1)}}(z) \widehat{\psi^{(2)}}(z):
$$

turns into the new bilocal fermionization formula [2] embedding the current into the real CAR algebra

$$
2 z \cdot \beta\left(\widehat{j}\left(z^{2}\right)\right)=: \widehat{\psi}(z) \widehat{\psi}(-z):
$$

(and a similar formula for the stress-energy tensor). We shall present the "bilocal gauge transformations" generated by this "embedded" current in Section 3.

2. The embedded stress-energy tensor consists of two pieces: the stress-energy tensor of the real Fermi theory, and an embedded current. Consequently, the embedded diffeomorphisms consist of a geometric flow and a bilocal gauge transformation. This is, in particular, true for the embedded Möbius transformations, which are known to arise as modular automorphisms in the vacuum state $[5,11]$. Therefore, the "modular mixing" $[7,15]$ of the Fermi field in multiple intervals finds its explanation as embedded Möbius transformations. Details of this will be presented in Section 4.

3. There is an analogous isomorphism between the real Fermi field in the Ramond representation and a pair of real Fermi fields, one in the Ramond, one in the vacuum representation. This yields another embedding of the current algebra into the Ramond representation of the real Fermi field, which turns out to be the "twisted" representation of the current [9]. The latter is defined by the quasifree state ${ }^{3}$ with two-point function

$$
\begin{aligned}
\omega_{t}(\widehat{j}(z) \hat{j}(w)) & =\frac{w+z}{2 \sqrt{w z}} \cdot \frac{1}{(w-z)^{2}} \\
\omega_{t}(j(x) j(y)) & =\frac{1+x y}{\sqrt{\left(1+x^{2}\right)\left(1+y^{2}\right)}} \cdot \frac{-1}{(x-y)^{2}},
\end{aligned}
$$

in the compact and non-compact picture, respectively. The twisted representation has lowest conformal energy $L_{0}=\frac{1}{16}$. Some aspects of this bilocal current will be discussed in Section 5 .

To conclude this section, we notice that Proposition 1 generalizes to an isomorphism between one real Fermi field and any number $n$ of real Fermi fields, exploiting the map $z \mapsto z^{n}$. Let us combine $n$ real Fermi fields into complex Fermi fields $\phi^{(k)}(k=1, \ldots, n)$ such that $\left(\phi^{(k)}(x)\right)^{*}=\phi^{(n+1-k)}(x)$. If $n$ is odd, $\phi^{\left(\frac{n+1}{2}\right)}$ is real.

${ }^{3} \mathrm{~A}$ state on a CAR or CCR algebra is called quasifree if it obeys Wick's theorem, i.e., all higher correlation functions are sums of products of two-point functions. 
PROPOSITION 2. The linear map

$$
\beta: \widehat{\phi^{(k)}}\left(z^{n}\right) \mapsto \frac{z^{1-k}}{n} \sum_{j=0}^{n-1} \omega^{(1-k) j} \cdot \widehat{\psi}\left(\omega^{j} z\right) \quad\left(z \in S^{1}, k=1, \ldots, n\right),
$$

where $\omega=e^{\frac{2 \pi i}{n}}$, induces an isomorphism $\beta: \mathrm{CAR}^{n}\left(S^{1}\right) \rightarrow \mathrm{CAR}\left(S^{1}\right)$ of CAR algebras, which preserves the vacuum state.

That this is a vacuum-preserving isomorphism, can again be verified as in the proof of Proposition 1, either by a direct computation of the vacuum expectation values, from which also the anti-commutation relations follow, or by noting that $\beta\left(\psi^{(k)}\left(z^{n}\right)\right)=\sum_{v \in \mathbb{Z}+\frac{1}{2}} \psi_{\frac{1}{2}-k+\left(v+\frac{1}{2}\right) n}\left(z^{n}\right)^{-v-\frac{1}{2}}$.

In particular, since non-abelian current algebras can be embedded into free Fermi theories with sufficiently many free Fermi fields, one obtains representations of all these theories in the Fock space of a single free Fermi field.

\section{Symmetries}

Throughout this section, we shall work exclusively in the compact picture, which drastically simplifies most formulae. The passage to the non-compact picture can always be made by the transformation laws $A(x)=\left(\frac{\sqrt{2}}{1-i x}\right)^{2 d} \widehat{A}(z)$ for fields of dimension $d$. We also suppress the symbol $\widehat{\cdot}$ in this section.

\subsection{GAUGE TRANSFORMATIONS}

The complex free Fermi field algebra is invariant under gauge transformations. The latter can be implemented by unitary Weyl operators:

$$
\begin{gathered}
\alpha_{f}(\phi(z)) \equiv e^{-i f(z)} \phi(z)=W(f) \phi(z) W(f)^{*}, \\
\alpha_{f}\left(\phi^{*}(z)\right) \equiv e^{i f(z)} \phi^{*}(z)=W(f) \phi^{*}(z) W(f)^{*},
\end{gathered}
$$

where $f: S^{1} \rightarrow \mathbb{R}$ is a smooth periodic function. In the vacuum representation, the Weyl operators are given by $W(f)=e^{i j(f)}, j(f)=\oint f(z) j(z) \mathrm{d} z / i$. The current $j(z)$ is contained in the Wick algebra of the complex Fermi field as

$$
j(z)=: \phi^{*}(z) \phi(z):=i: \psi^{(1)}(z) \psi^{(2)}(z): .
$$

It satisfies the CCR algebra

$$
[j(f), j(g)]=2 \pi i \oint f(z) \partial_{z} g(z) \mathrm{d} z,
$$

and its vacuum two-point function is

$$
\omega(j(z) j(w))=\frac{1}{(z-w)^{2}} .
$$


The current algebra defines a free Bose quantum field of its own. The CCR algebra possesses automorphisms

$$
\rho_{\lambda}(j(z))=j(z)+\lambda(z),
$$

where $z \lambda(z)$ is a smooth real function. It is well known, that states $\omega_{\lambda}=\omega_{0} \circ \rho_{\lambda}$ are charged states of charge $q=\frac{1}{2 \pi} \int \lambda(z) \mathrm{d} z$, and these states are ground states for the conformal Hamiltonian $L_{0}$ if $\lambda(z)=\frac{q}{z}$. In this case, we denote $\rho_{\lambda}=\rho^{q}$ :

$$
\rho^{q}(j(z))=j(z)+\frac{q}{z} .
$$

Because the Wick product is defined by the subtraction of vacuum expectation values, and the isomorphism $\beta$ respects the vacuum state, the latter extends to the Wick algebra of the complex Fermi field. In particular, we can "embed" the current into the real Fermi algebra. A straightforward computation gives rise to

COROLLARY 1. The embedded current is

$$
\beta\left(j\left(z^{2}\right)\right)=\frac{1}{2 z}: \psi(z) \psi(-z): .
$$

We may also write this in terms of Fourier modes

$$
j(z)=\sum_{n \in \mathbb{Z}} j_{n} z^{-n-1},
$$

where $j_{n}^{*}=j_{-n}$ satisfy the CCR $\left[j_{m}, j_{n}\right]=m \delta_{m+n, 0}$. Then

$$
\beta\left(j\left(z^{2}\right)\right)=\frac{1}{2 z} \sum_{m, k \in \mathbb{Z}+\frac{1}{2}}: \psi_{m} \psi_{k}:(-1)^{-k-\frac{1}{2}} z^{-m-k-1} .
$$

If $N \in \mathbb{Z}$ is odd, the sum $\sum_{k \in \mathbb{Z}+\frac{1}{2}}: \psi_{N-k} \psi_{k}:(-1)^{-k-\frac{1}{2}}=\sum_{k \in \mathbb{Z}+\frac{1}{2}}: \psi_{k} \psi_{N-k}$ : $(-1)^{k-N-\frac{1}{2}}$ vanishes by virtue of the CAR, because $(-1)^{k-N-\frac{1}{2}}=(-1)^{-k-\frac{1}{2}}$. Thus, the sum contains only odd powers $z^{-N-1}=z^{-2 n-1}$, and the expansion in $z^{2}$ yields

$$
\beta\left(j_{n}\right)=\sum_{\nu=0}^{\infty}(-1)^{n+v+1} \psi_{n-v-\frac{1}{2}} \psi_{n+v+\frac{1}{2}} .
$$

The following corollary gives the action of the embedded gauge transformations on the real free Fermi field:

COROLLARY 2. The embedded gauge transformations $\beta(W(f)) A \beta(W(f))^{*}=\beta$ 。 $\alpha_{f} \circ \beta^{-1}(A)$ act on the real Fermi field by

$$
\beta(W(f)) \psi(z) \beta(W(f))^{*}=\cos f\left(z^{2}\right) \cdot \psi(z)+\sin f\left(z^{2}\right) \cdot \psi(-z) .
$$

The characteristic feature is the bilocal "mixing" of $\psi(z)$ and $\psi(-z)$, reflecting the non-locality of the isomorphism $\beta$. 


\subsection{DIFFEOMORPHISMS}

The real and complex Fermi field algebras are invariant under diffeomorphisms $\gamma$ : $S^{1} \rightarrow S^{1}$, implemented by unitary operators

$$
V(\gamma) \psi(z) V(\gamma)^{*}=\sqrt{\gamma^{\prime}(z)} \cdot \psi(\gamma(z)) .
$$

The unitary implementers of one-parameter groups of diffeomorphisms are given by

$$
V\left(\gamma_{t}\right)=e^{i t T(f)} \equiv e^{i t \oint f(z) T(z) \mathrm{d} z}
$$

where if $(z) / z \in \mathbb{R}$. The infinitesimal diffeomorphisms given by derivations

$$
\delta_{f}(\psi(z)) \equiv i[T(f), \psi(z)]=\left(-f(z) \partial_{z}-\frac{1}{2} f^{\prime}(z)\right) \psi(z)
$$

integrate to one-parameter groups of finite diffeomorphisms via $\partial_{t} \gamma_{t}(z)=-f\left(\gamma_{t}(z)\right)$.

The stress-energy tensor of the real Fermi field is

$$
T^{c=\frac{1}{2}}(z)=\frac{-1}{4 \pi}: \psi \partial \psi:(z)=\frac{-1}{8 \pi}: \psi \stackrel{\leftrightarrow}{\partial} \psi:(z),
$$

the one of the complex Fermi field is

$$
T^{c=1}(z)=\frac{-1}{4 \pi}: \psi^{(1)} \partial \psi^{(1)}:(z)+\frac{-1}{4 \pi}: \psi^{(2)} \partial \psi^{(2)}:(z)=\frac{-1}{4 \pi}: \phi^{*} \stackrel{\leftrightarrow}{\partial} \phi:(z) .
$$

The current algebra is also diffeomorphism invariant,

$$
V(\gamma) j(z) V(\gamma)^{*}=\gamma^{\prime}(z) \cdot j(\gamma(z)),
$$

with the stress-energy tensor given by

$$
T^{\mathrm{curr}}(z)=\frac{1}{4 \pi}: j^{2}:(z) .
$$

If the current is expressed by the complex Fermi field as above, then this coincides with $T^{c=1}$ :

$$
\frac{1}{4 \pi}: j^{2}:(z)=\frac{-1}{4 \pi}: \phi^{*} \stackrel{\leftrightarrow}{\partial} \phi:(z) .
$$

Our isomorphism $\beta$ embeds the stress-energy tensor $T^{c=1}$ into the real free Fermi theory. Again, the computation is straightforward. The result is

COROLLARY 3. The embedded complex stress-energy tensor is

$$
\beta\left(T^{c=1}\left(z^{2}\right)\right)=-\frac{1}{8 \pi z^{2}} \beta\left(j\left(z^{2}\right)\right)+\frac{1}{4 z^{2}}\left(T^{c=\frac{1}{2}}(z)+T^{c=\frac{1}{2}}(-z)\right) .
$$


The embedded infinitesimal diffeomorphisms $i\left[\beta\left(T^{c=1}(f)\right), A\right]=\beta \circ \delta_{f} \circ \beta^{-1}(A)$ act on the real Fermi field by

$$
\begin{aligned}
i\left[\beta\left(T^{c=1}(f)\right), \psi(z)\right]= & \left(-\frac{1}{2 z} f\left(z^{2}\right) \partial_{z}-\frac{1}{2} f^{\prime}\left(z^{2}\right)\right) \psi(z) \\
& +\frac{1}{4 z^{2}} f\left(z^{2}\right)(\psi(z)-\psi(-z)) .
\end{aligned}
$$

Again, we have a mixing of $\psi(z)$ and $\psi(-z)$, due to the first contribution in (3.7), on top of a geometric flow due to the second term.

The contribution from the current in (3.7) can be removed by composition with a charged automorphism of the current algebra. Thus, the relation (3.7) may also be written as

$$
\beta \circ \rho^{\frac{1}{4}}\left(T^{c=1}\left(z^{2}\right)\right)=\frac{1}{4 z^{2}}\left(T^{c=\frac{1}{2}}(z)+T^{c=\frac{1}{2}}(-z)\right)+\frac{1}{64 \pi z^{4}} .
$$

One may embed conversely the stress-energy tensor of the real Fermi field into the algebra of the complex Fermi field. Because of the form (2.7) of the inverse isomorphism $\beta^{-1}$, this will involve terms : $\phi \partial \phi$ : and its conjugate, so that the embedded diffeomorphisms of the real Fermi field will generate transformations involving the charge conjugation $\phi \leftrightarrow \phi^{*}$.

COROLLARY 4. The embedded real stress-energy tensor is

$$
\beta^{-1}\left(T^{c=\frac{1}{2}}(z)\right)=-2 z^{2} T^{c=1}\left(z^{2}\right)+\frac{1}{4 \pi} j\left(z^{2}\right)-\frac{z}{2 \pi}\left(: \phi \partial \phi:\left(z^{2}\right)+z^{2}: \phi^{*} \partial \phi^{*}:\left(z^{2}\right)\right) .
$$

The embedded infinitesimal diffeomorphisms $i\left[\beta^{-1}\left(T^{c=\frac{1}{2}}(f)\right), A\right]=\beta^{-1} \circ \delta_{f} \circ \beta(A)$ act on the complex Fermi field by

$$
\begin{aligned}
i\left[\beta^{-1}\left(T^{c=\frac{1}{2}}(f)\right), \phi\left(z^{2}\right)\right]= & -f_{-}\left(z^{2}\right) \phi^{\prime}\left(z^{2}\right)-\frac{1}{2} f_{-}^{\prime}\left(z^{2}\right) \phi\left(z^{2}\right)+\frac{1}{4 z^{2}} f_{-}\left(z^{2}\right) \phi\left(z^{2}\right) \\
& -z^{2} f_{+}\left(z^{2}\right) \phi^{* \prime}\left(z^{2}\right)-\frac{z^{2}}{2} f_{+}^{\prime}\left(z^{2}\right) \phi^{*}\left(z^{2}\right)-\frac{1}{2} f_{+}\left(z^{2}\right) \phi^{*}\left(z^{2}\right)
\end{aligned}
$$

where $f_{-}\left(z^{2}\right)=z(f(z)-f(-z)), f_{+}\left(z^{2}\right)=f(z)+f(-z)$.

Let us also introduce the Fourier modes, $\widehat{T}(z)=\sum_{n \in \mathbb{Z}} T_{n} z^{-n-2}$. The standard Virasoro generators are related to the Fourier modes $T_{n}$ by $L_{n}=2 \pi T_{n}$. Then the relations (3.7) and (3.9) are equivalent to

$$
\beta\left(L_{n}^{c=1}\right)=-\frac{1}{4} \beta\left(j_{n}\right)+\frac{1}{2} L_{2 n}^{c=\frac{1}{2}}, \quad \beta \circ \rho^{\frac{1}{4}}\left(L_{n}^{c=1}\right)=\frac{1}{2} L_{2 n}^{c=\frac{1}{2}}+\frac{1}{32} \delta_{n, 0},
$$

where $\rho^{q}$ is the charged automorphism (3.3) extended to the stress-energy tensor contained in the Wick algebra of the current. 
The latter expression is well known as the embedding of the infinitesimal "twodiffeomorphisms" (i.e., the diffeomorphisms of $z$ induced from those of the variable $z^{2}$ ) as a subalgebra of the Virasoro algebra. Note that the application of $\rho^{\frac{1}{4}}$ to the embedded current (3.4) exactly "undoes" the subtraction of the vacuum expectation in the definition as a Wick product. Indeed, the subtraction is not necessary in order to obtain a well-defined current, because the points are split anyway. But the "unsubtracted" current has a non-vanishing vacuum expectation value, i.e., the corresponding state is a state of charge $q=\frac{1}{4}$.

In contrast, the first expression in (3.12) involves also the modes of the current, which may in turn be expressed as before by the real Fermi modes. The present form emphasizes that the embedded diffeomorphisms come along with embedded gauge transformation, i.e., a mixing of $\psi(z)$ and $\psi(-z)$, as described by Corollary 2 .

\section{Modular Theory}

This brings us to the second issue, which finds a simple explanation by Proposition 1.

"Modular automorphisms" are a one-parameter group of automorphisms of a von Neumann algebra $M$ in a faithful normal state $\omega$, canonically associated with the pair $(M, \omega)$. It is of particular interest in quantum physics, because the state is automatically a thermal equilibrium state for the time evolution given by the modular automorphisms ("modular dynamics") [13], and because the modular automorphisms of local algebras for certain simple spacetime regions coincide with Lorentz or conformal transformations [3,5,11]. The latter fact has been exploited $[4,12,14]$ to show that the full content of a quantum field theory can be encoded in the vacuum state and a small finite number of von Neumann algebras "in suitable modular position", such that their modular groups generate all spacetime symmetries, and all local algebras can be identified as intersections of the transforms of the initial algebras.

In the case of chiral conformal theories, the modular automorphisms for the algebras of observables in an interval $I$ coincide with the one-parameter subgroup of Möbius transformations that preserve the interval [5,11], namely the dilations $\lambda_{-2 \pi t}: x \mapsto e^{-2 \pi t} x$ if $I=\mathbb{R}_{+}$in the non-compact picture:

$$
\sigma_{t}(\psi(x))=U\left(\lambda_{-2 \pi t}\right) \psi(x) U\left(\lambda_{-2 \pi t}\right)^{*}=e^{-\pi t} \psi\left(e^{-2 \pi t} x\right) \quad\left(I=\mathbb{R}_{+}\right) .
$$

For every other interval, the modular automorphisms are obtained by conjugation with a Möbius transformation that maps $I$ onto $\mathbb{R}_{+}$. Note that Möbius transformations intertwine the modular automorphisms, because they preserve the vacuum state.

The modular automorphisms of the von Neumann algebra of the Fermi field in multiple intervals in the vacuum state was found in [7], and elaborated in [15]. It 
is given by a combination of a geometric flow and a "mixing" of field operators in the different intervals, which we describe in the following.

An $n$-interval is the union $\widehat{E}=\cup_{k} \widehat{I}_{k}$ of $n$ open intervals with disjoint closure in $S^{1}$. It is called "symmetric" if $\widehat{I}_{k}$ are the $n$th roots of an interval $\widehat{I} \subset S^{1}$. We may assume that the point $z=-1$ is not in the closure of $\widehat{E}$, i.e., the Cayley preimage $E \subset \mathbb{R}$ is bounded. Otherwise, we may first apply a Möbius transformation. Because Möbius transformations intertwine the modular automorphisms, the modular flow would be a Möbius conjugate of the following.

Let the intervals be given by $\widehat{I}_{k}=\left(u_{k}, v_{k}\right) \subset S^{1}$, and define the function $X$ by

$$
X(z):=-\prod_{k} \frac{x-a_{k}}{x-b_{k}} \equiv-\prod_{k} \frac{1+v_{k}}{1+u_{k}} \cdot \prod_{k} \frac{z-u_{k}}{z-v_{k}} .
$$

Here, $x, a_{k}, b_{k} \in \mathbb{R}$ are the Cayley pre-images of $z, u_{k}, v_{k} \in S^{1}$.

This function maps each of the intervals monotonously onto $\mathbb{R}_{+}$, so that every $X \in \mathbb{R}_{+}$has exactly $n$ pre-images $z_{k}(X)$, one in each interval. Then the geometric flow is

$$
\delta_{t}\left(z_{k}(X)\right)=z_{k}\left(e^{-2 \pi t} X\right) .
$$

The modular automorphism group acts on the Fermi field by

$$
\sqrt{z_{k}^{\prime}(X)} \cdot \sigma_{t}\left(\widehat{\psi}\left(z_{k}\right)\right)=\sum_{j} O_{k j}(t, X) \sqrt{z_{j}^{\prime}\left(e^{-2 \pi t} X\right)} \cdot \widehat{\psi}\left(\delta_{t}\left(z_{j}\right)\right)
$$

where $z_{k}^{\prime} \equiv \mathrm{d} z_{k}(X) / \mathrm{d} X$. The orthogonal mixing matrix $O(t, X) \in S O(n)$ is a cocyle $O(t+s, X)=O(t, X) O\left(s, e^{-2 \pi t} X\right)$, solving the differential equation ${ }^{4}$

$$
\partial_{t} O(t, X)=O(t, X) K\left(e^{-2 \pi t} X\right)
$$

where

$$
K(X)_{k j}=2 \pi \frac{\sqrt{z_{k}^{\prime}(X) z_{j}^{\prime}(X)}}{z_{k}(X)-z_{j}(X)} \quad(k \neq j), \quad K(x)_{k k}=0 .
$$

The solution is a coboundary

$$
O(t, X)=O(X)^{T} \cdot O\left(e^{-2 \pi t} X\right)
$$

where $O(X)$ is the anti-path-ordered exponential

$$
O(X)=\bar{P} \exp \left(-\frac{1}{2 \pi} \int_{X_{0}}^{X} K\left(X^{\prime}\right) \mathrm{d} X^{\prime}\right) .
$$

\footnotetext{
${ }^{4}$ This equation is incorrectly displayed in [15] as $\partial_{t} O(t)=K(t) O(t)$. The error is due to a change of notation between [7] and [15]. Namely, [7] write $\sigma_{t}\left(\psi\left(e^{+2 \pi t} X\right)\right)=O_{\mathrm{CH}}(t, X) \psi(X)$, so that by comparison with (4.3), $O(t, X)=O_{\mathrm{CH}}\left(t, e^{-2 \pi t} X\right)=O_{\mathrm{CH}}(-t, X)^{-1}$. The confusion arises because both articles suppress the $X$-dependence of their mixing matrices. By [7], the differential equation $\partial_{t} O_{\mathrm{CH}}(t, X)=K\left(e^{+2 \pi t} X\right) O_{\mathrm{CH}}(t, X)$ holds. This implies the correct equation (4.4).
} 
It follows that the modular mixing is "diagonalized" by the position-dependent orthogonal matrix $O(X)$ : Let

$$
\chi_{k}(X):=\sum_{j} O_{k j}(X) \sqrt{z_{j}^{\prime}(X)} \cdot \widehat{\psi}\left(z_{j}(X)\right) .
$$

Then (4.3) becomes

$$
\sigma_{t}\left(\chi_{k}(X)\right)=e^{-\pi t} \chi_{k}\left(e^{-2 \pi t} X\right) .
$$

That is, by diagonalizing the modular mixing and reparametrizing $z_{k}=z_{k}(X)$, one recovers the modular automorphisms of $n$ independent Fermi fields in $\mathbb{R}_{+}$.

To make the connection with Proposition 2, let us specialize to symmetric $n$-intervals. In this case, the related points in $\widehat{I}_{k}$ are given by $z_{k}=\omega^{k} z$, where $\omega=$ $e^{\frac{2 \pi i}{n}}$. It follows that $-\frac{1}{2 \pi} K(X) \mathrm{d} X=\frac{K}{z} \mathrm{~d} z$, where $z=e^{i \varphi}$ and $K$ is the constant antisymmetric matrix with non-diagonal entries

$$
K_{k j}=-\frac{\omega^{\frac{k+j}{2}}}{\omega^{k}-\omega^{j}},
$$

hence

$$
O(X)=\exp \left(K \int_{1}^{z} \frac{\mathrm{d} w}{w}\right)=z^{K} .
$$

LEMMA. The matrix $K$ has integer-spaced spectrum $\frac{1-n}{2}, \ldots, \frac{n-1}{2}$. It is diagonalized by the unitary matrix $\frac{1}{\sqrt{n}} B, B_{k j}=\omega^{\left(\frac{1}{2}-k\right) j}$, i.e., $B K=M B$ where $M$ is the diagonal matrix with entries $m_{k k}=\frac{n+1}{2}-k(k=1, \ldots, n)$.

Proof. By direct computation (using $\omega^{n}=1$ )

$$
\begin{aligned}
\sum_{j=1, \ldots, n, j \neq l} B_{k j} K_{j l} & =-\sum_{j=l+1}^{n+l-1} \frac{\omega^{\left(\frac{1}{2}-k\right) j} \omega^{\frac{j+l}{2}}}{\omega^{j}-\omega^{l}}=-\omega^{\left(\frac{1}{2}-k\right) l} \sum_{j=1}^{n-1} \frac{\omega^{\left(\frac{1}{2}-k\right) j} \omega^{\frac{j+2 l}{2}}}{\left(\omega^{j}-1\right) \omega^{l}} \\
& \equiv-B_{k l} \sum_{j=1}^{n-1} \frac{\omega^{(1-k) j}}{\left(\omega^{j}-1\right)}=-B_{k l} \cdot \frac{1}{2} \sum_{j=1}^{n-1}\left(\frac{\omega^{(1-k) j}}{\left(\omega^{j}-1\right)}+\frac{\omega^{(1-k)(n-j)}}{\left(\omega^{n-j}-1\right)}\right) \\
& \equiv-B_{k l} \cdot \frac{1}{2} \sum_{j=1}^{n-1} \frac{\omega^{(1-k) j}-\omega^{k j}}{\left(\omega^{j}-1\right)}=B_{k l} \cdot \frac{1}{2} \sum_{j=1}^{n-1} \sum_{\nu=1-k}^{k-1} \omega^{j v} \\
& =B_{k l} \cdot \frac{1}{2} \sum_{\nu=1-k}^{k-1}\left(n \delta_{v, 0}-1\right)=B_{k l} \cdot \frac{1}{2}(n-2 k+1)=m_{k k} \cdot B_{k l} .
\end{aligned}
$$

In the first line, we have used the invariance under $j \rightarrow j+n$ and shifted the summation index by $l$. In the second line, we have symmetrized the sum w.r.t. $j \leftrightarrow$ $n-j$. In the third line, we have cancelled the denominator, and in the last line, we have used $\sum_{j=0}^{n} \omega^{j v}=n \delta_{\nu, 0}$. 
PROPOSITION 3. The isomorphism given in Proposition 2 intertwines the modular group of $n$ free Fermi fields in an interval $\widehat{I} \subset S^{1}$ with the modular group of a single free Fermi field in the symmetric n-interval $\widehat{E}=\sqrt[n]{\widehat{I}}$.

Proof. By virtue of the lemma, (4.6) can be written as

$$
\sum_{j} B_{k j} \chi_{j}(X)=\frac{z^{1-k}}{n} \sum_{j} \omega^{(1-k) j} \widehat{\psi}\left(\omega^{j} z\right)
$$

The right-hand side is precisely $\beta\left(\widehat{\phi^{(k)}}\left(z^{n}\right)\right)$ according to Proposition 2. We claim that $X\left(z^{n}\right)$ is the composition of the Cayley transform $z^{n} \mapsto \frac{z^{n}-1}{i\left(z^{n}+1\right)}$ that maps $\widehat{I}$ onto $I$, with a Möbius transformation that maps $I$ onto $\mathbb{R}_{+}$. Both Möbius transformations and the isomorphism $\beta$ preserve the vacuum state and hence intertwine the respective modular automorphisms. Knowing the modular automorphisms of $\widehat{\phi^{(k)}}\left(z^{n}\right)$ for $z^{n} \in \widehat{I}$, it follows that the modular automorphisms of $\chi_{j}(X)$ are given by the dilations $X \rightarrow e^{-2 \pi t} X$ on $\mathbb{R}_{+}$. This explains the modular automorphisms (4.7) and hence also (4.3). It only remains to verify the claim that the function $X$ given by (4.2) is indeed of the said form. But, in the symmetric case one may use identities like $\prod_{k}\left(z-w_{k}\right)=z^{n}-w^{n}$ to find

$$
X=-\frac{(-1)^{n}-v^{n}}{(-1)^{n}-u^{n}} \cdot \frac{z^{n}-u^{n}}{z^{n}-v^{n}}
$$

which is indeed a Möbius transform of $\frac{z^{n}-1}{i\left(z^{n}+1\right)}$.

It should be noted that by what has been said, (4.6) diagonalizes the modular flow only inside the $n$-interval $\left(z_{k} \in \widehat{I_{k}}, z^{n} \in \widehat{I}\right)$, whereas the isomorphism $\beta$ manifestly extends to the entire circle. This reflects the fact that the graded commutant (denoted by ${ }^{c}$ ) of the Fermi field algebra in an $n$-interval equals the Fermi field algebra in the complement of the $n$-interval:

$$
\pi_{0}(\operatorname{CAR}(\widehat{E}))^{c}=\pi_{0}\left(\operatorname{CAR}\left(S^{1} \backslash \widehat{E}\right)\right)^{\prime \prime}, \quad \pi_{0}\left(\operatorname{CAR}^{n}(\widehat{I})\right)^{c}=\pi_{0}\left(\operatorname{CAR}^{n}\left(S^{1} \backslash \widehat{I}\right)\right)^{\prime \prime} .
$$

By Modular Theory, the modular flow of the graded commutant is given by the inverse of the modular flow of the original algebra. Thus, the same orthogonal matrix $O(X)$ also diagonalizes the modular flow on the complements.

Finally, our result answers a question raised by M. Bischoff and Y. Tanimoto: If $\widehat{E}$ is a symmetric $n$-interval, and $\widehat{F} \subset \widehat{E}$ is a symmetric subinterval with common upper limits of the intervals, let $M:=\pi_{0}(\operatorname{CAR}(\widehat{E}))^{\prime \prime}$ and $N:=\pi_{0}(\operatorname{CAR}(\widehat{F}))^{\prime \prime}$. Then, the data $\left(N_{0}, N_{1}, N_{2}, \Omega\right)$ with $N_{0}=M^{c}, N_{1}=N, N_{2}=N^{c} \cap M$ form a " + half-sided modular factorization" in the sense of [12, Thm. 1.2] (actually, a graded generalization thereof: one has $N_{i} \subset N_{i+1}^{c} \bmod 3$, and the modular automorphisms $\sigma_{-t}$ of the larger algebra map the smaller algebra into itself for $t \geq 0$ ). By the main theorem of [12], they therefore define a (graded local) conformal quantum field theory 
such that its local algebras are given by $A\left(\mathbb{R}_{+}\right)=M, A\left(\mathbb{R}_{+}+1\right)=N$, and the Möbius transformations are generated by the modular groups of the three algebras.

COROLLARY 5. The conformal QFT obtained from the graded +-hsm factorization $\left(N, M^{c}, N^{c} \cap M, \Omega\right)$ is isomorphic to the free Fermi theory $\operatorname{CAR}^{n}\left(S^{1}\right)$ of $n$ free Fermi fields.

Namely, the isomorphism is just given by $\beta^{-1}$, which maps $M$ onto $\pi_{0}\left(\operatorname{CAR}^{n}(\widehat{I})\right)^{\prime \prime}$ and $N$ onto $\pi_{0}\left(\operatorname{CAR}^{n}(\widehat{J})\right)^{\prime \prime}$ (where $\widehat{E}=\sqrt[n]{\widehat{I}}, \widehat{F}=\sqrt[n]{\widehat{J}}$ ), combined with a Möbius transformation which maps $I$ onto $\mathbb{R}_{+}$and $J$ onto $\mathbb{R}_{+}+1$. Both isomorphisms intertwine the respective vacuum modular groups.

\section{The Ramond Case}

The real free Fermi field possesses another faithful representation of positive energy: the Ramond representation induced (by the GNS construction) from the quasifree ground state with two-point function

$$
\omega_{R}(\psi(x) \psi(y))=\frac{1+x y}{\sqrt{1+x^{2}} \sqrt{1+y^{2}}} \cdot \frac{-i}{x-y-i \varepsilon} .
$$

In the compact picture on $S^{1} \backslash\{-1\}$, this is

$$
\omega_{R}(\widehat{\psi}(z) \widehat{\psi}(w))=\frac{z+w}{2 \sqrt{z w}} \cdot \frac{1}{z-w} .
$$

Obviously, in the Ramond representation, $\pi_{R}(\widehat{\psi}(z))$ has a cut at $z=-1$ and extends anti-periodically to $S^{1}$. It is therefore convenient to introduce the Ramond field

$$
\psi_{R}(z):=\sqrt{z} \cdot \pi_{R}(\widehat{\psi}(z))
$$

which extends periodically to $S^{1}$ with Fourier representation

$$
\psi_{R}(z)=\sum_{n \in \mathbb{Z}} \psi_{R, n} z^{-n},
$$

where $\psi_{R, n}^{*}=\psi_{R,-n}$ satisfy the CAR $\left\{\psi_{R, n}, \psi_{R, m}\right\}=\delta_{n+m, 0}$. In particular, this field has a zero mode with $2 \psi_{R, 0}{ }^{2}=1$.

Because we are going to consider the field both in the vacuum and in the Ramond representation, we shall below write the field in the vacuum representation as $\psi_{0}(z):=\pi_{0}(\widehat{\psi}(z))$, and write no subscript when the field is understood algebraically.

The modular theory of the Ramond field is not known. We therefore lack a rationale to expect a similar isomorphism as in Proposition 1, which would explain a modular mixing as in Proposition 3. 
Nevertheless, one can prove along the same lines as in Proposition 1 the following

\section{PROPOSITION 4. The linear map}

$$
\begin{aligned}
& \beta_{R}: \psi_{R}^{(1)}\left(z^{2}\right) \equiv \psi_{R}\left(z^{2}\right) \otimes^{t} \mathbf{1} \\
& \mapsto \frac{1}{2}\left(\psi_{R}(z)+\psi_{R}(-z)\right), \\
& \psi_{0}^{(2)}\left(z^{2}\right) \equiv \mathbf{1} \otimes^{t} \psi_{0}\left(z^{2}\right) \mapsto \frac{1}{2 z}\left(\psi_{R}(z)-\psi_{R}(-z)\right)
\end{aligned}
$$

for $z \in S^{1}$, induces an isomorphism of CAR algebras $\beta: \pi_{R}\left(\operatorname{CAR}\left(S^{1}\right)\right) \otimes^{t} \pi_{0}\left(\operatorname{CAR}\left(S^{1}\right)\right)$ $\rightarrow \pi_{R}\left(\operatorname{CAR}\left(S^{1}\right)\right)$, such that $\omega_{R} \circ \beta_{R}=\omega_{R} \otimes \omega_{0}$.

Namely, in terms of the Fourier modes, the right-hand-sides equal, respectively, $\sum_{n \in \mathbb{Z}} \psi_{R, 2 n} z^{-2 n}$ and $\sum_{n \in \mathbb{Z}} \psi_{R, 2 n+1} z^{-2 n-2}$, from which the correct commutation relations and two-point functions follow.

The fact that the current (3.1) $j(x)=i: \psi^{(1)}(x) \psi^{(2)}(x)$ : satisfies the CCR (3.2) is purely algebraic, and therefore independent of the representation. Taking $\psi^{(1)}$ in the Ramond representation and $\psi^{(2)}$ in the vacuum representation, the isomorphism (5.1) embeds the resulting current into the Ramond algebra $\pi_{R}\left(\operatorname{CAR}\left(S^{1}\right)\right)$. The result is (in the compact picture)

$$
\beta_{R}\left(\widehat{j}\left(z^{2}\right)\right)=\frac{1}{2 i z^{2}} \cdot: \psi_{R}(z) \psi_{R}(-z):_{R}
$$

where $: \because R_{R}$ stands for the Wick product defined with the subtraction of the Ramond expectation value (which is zero in the case at hand). The right-hand side is the formula given in [1]. The new aspect here is that it arises from an underlying isomorphism of CAR algebras.

The embedded current (5.2) changes sign under $z \mapsto-z$, hence is anti-periodic in the variable $z^{2}$. In other words, we find the current in a representation that extends anti-periodically to the circle. Indeed, this representation of the current is known as the "twisted" representation [1,9] obtained from the quasifree state with twopoint function given by (2.12). This can be established by evaluating the embedded current in the Ramond state. We find by direct computation

$$
\omega_{R}\left(\beta_{R}\left(\widehat{j}\left(z^{2}\right)\right)\right)=0, \quad \omega_{R}\left(\beta_{R}\left(\widehat{j}\left(z^{2}\right)\right) \beta_{R}\left(\widehat{j}\left(w^{2}\right)\right)\right)=\frac{z^{2}+w^{2}}{2 z w\left(z^{2}-w^{2}\right)^{2}} .
$$

The state $\omega_{R} \circ \beta_{R}$ restricted to the current is again quasifree. Thus, the embedding of the current via the isomorphism (5.1) produces the twisted representation of the CCR algebra:

$$
\omega_{R} \circ \beta_{R}=\omega_{t} .
$$


Finally, we have computed the stress-energy tensor using the bosonic formula $T^{\mathrm{curr}}(x)=\frac{1}{4 \pi}: j(x)^{2}$.. We find the same formula as (3.9):

$$
\beta_{R}\left(T^{\text {curr }}\left(z^{2}\right)\right)=\frac{1}{4 z^{2}}\left(\pi_{R}\left(T^{c=\frac{1}{2}}(z)\right)+\pi_{R}\left(T^{c=\frac{1}{2}}(-z)\right)\right)+\frac{1}{64 \pi z^{4}} .
$$

(In deriving this equation, care must be taken of the fact that due to the different subtractions, one has $: \widehat{\psi}(z) \partial_{z} \widehat{\psi}(z):_{R}=: \widehat{\psi}(z) \partial_{z} \widehat{\psi}(z):_{0}+\frac{1}{8 z^{2}}$.) Since $L_{0}^{c=\frac{1}{2}}$ has eigenvalue $h_{R}=\frac{1}{16}$ in the Ramond state, we conclude that $\omega_{R}\left(\beta_{R}\left(L_{0}^{\text {curr }}\right)\right)=\frac{1}{16}$, in agreement with the ground state energy in the twisted sector of the current.

\section{Conclusion}

We have described a multilocal isomorphism of the CAR algebra of a real chiral Fermi field with the CAR algebra of any number $n$ of such fields. The isomorphism preserves the vacuum state. This has two consequences:

(1) The isomorphism extends to Wick products, and therefore allows to "embed" the generators of local symmetries (gauge transformations or diffeomorphisms) of one theory into the other. We have explicitly displayed the "embedded" symmetries and their multilocal features in the case $n=2$. A characteristic feature is that the gauge transformations of the complex Fermi field, embedded into the algebra of the real Fermi field, generate a mixing of Fermi fields at different points; and that the embedded diffeomorphisms consist of a mixing on top of a geometric transformation.

(2) A similar mixing was found earlier to occur in the modular automorphisms of the chiral Fermi algebra in multiple intervals. We have demonstrated that this modular mixing is a special case of the mixing going with the embedded diffeomorphisms, when the latter arise as modular automorphisms for a single interval, i.e., certain one-parameter groups of Möbius transformations. However, as stated in (1), the embedded diffeomorphisms are not restricted to Möbius transformations, and, in view of the fact that the vacuum state is not diffeomorphism invariant, are not necessarily related to modular theory.

Finally, we observed that a similar isomorphism exists in the Ramond representation, and we have computed its restriction to the subalgebra of the current and the stress-energy tensor.

\section{Acknowledgements}

This work was supported in part by the German Research Foundation [Deutsche Forschungsgemeinschaft (DFG)] through the Institutional Strategy of the University of Göttingen, and by the DFG Research Training School 1493 "Mathematical Structures in Modern Quantum Physics". KHR is grateful to I. Anguelova 
for interesting discussions, which triggered this work, and to Y. Tanimoto and M. Bischoff, who raised interesting questions.

Open Access This article is distributed under the terms of the Creative Commons Attribution License which permits any use, distribution, and reproduction in any medium, provided the original author(s) and the source are credited.

\section{References}

1. Anguelova, I.: Boson-fermion correspondence of type B and twisted vertex algebras (2011). arXiv:1112.3913

2. Anguelova, I.: Twisted vertex algebras, bicharacter construction and boson-fermion correspondences (2012). arXiv:1206.4026

3. Bisognano, J.J., Wichmann, E.H.: On the duality condition for quantum fields. J. Math. Phys. 17, 303-321 (1976)

4. Borchers, H.-J.: The CPT theorem in two-dimensional theories of local observables. Commun. Math. Phys. 143, 315-322 (1992)

5. Brunetti, R., Guido, D., Longo, R.: Modular structure and duality in conformal quantum field theory. Commun. Math. Phys. 156, 201-219 (1993)

6. Carey, A.L., Ruijsenaars, S.N.M., Wright, J.D.: The massless Thirring model: positivity of Klaiber's $N$-point functions. Commun. Math. Phys. 99, 347-364 (1985)

7. Casini, H., Huerta, M.: Reduced density matrix and internal dynamics for multicomponent regions. Class. Quant. Gravity 26, 185005 (2009)

8. Date, E., Jimbo, M., Kashiwara, M., Miwa, T.: Transformation groups for soliton equations. 4. A new hierarchy of soliton equations of KP type. Physica D4, 343-365 (1982)

9. Fredenhagen, K.: Superselection sectors with infinite statistical dimension (1994, preprint DESY-94-071)

10. Fuchs, J.: Affine Lie Algebras and Quantum Groups. Cambridge University Press, London (1992)

11. Fröhlich, J., Gabbiani, F.: Operator algebras and conformal field theory. Commun. Math. Phys. 155, 569-640 (1993)

12. Guido, D., Longo, R., Wiesbrock, H.-W.: Extensions of conformal nets and superselection structures. Commun. Math. Phys. 192, 217-244 (1998)

13. Haag, R., Hugenholtz, N., Winnink, M.: On the equilibrium states in quantum statistical mechanics. Commun. Math. Phys. 5, 215-236 (1967)

14. Kähler, R., Wiesbrock, H.-W.: Modular theory and the reconstruction of four-dimensional quantum field theories. J. Math. Phys. 42, 74-86 (2001)

15. Longo, R., Martinetti, P., Rehren, K.-H.: Geometric modular action for disjoint intervals and boundary conformal field theory. Rev. Math. Phys. 22, 331-354 (2010)

16. Mandelstam, S.: Soliton operators for the quantized sine-Gordon equation. Phys. Rev. D11, 3026 (1975)

17. Stone, M. (ed): Bosonization. World Scientific, Singapore (1994) 